Glauco Sanga Daniel Baggioni

Les dynamiques linguistiques de la société italienne (18611980) : de la naissance de l'italien populaire à la diffusion des ethnicismes linguistiques

In: Langages, $15 \mathrm{e}$ année, $n^{\circ} 61,1981$. pp. 93-115.

Citer ce document / Cite this document :

Sanga Glauco, Baggioni Daniel. Les dynamiques linguistiques de la société italienne (1861-1980) : de la naissance de l'italien populaire à la diffusion des ethnicismes linguistiques. In: Langages, 15e année, n61, 1981. pp. 93-115.

doi : $10.3406 /$ lgge.1981.1870

http://www.persee.fr/web/revues/home/prescript/article/lgge_0458-726X_1981_num_15_61_1870

BY: +2 creative 
Glauco SANGA

Université de Pavie

\section{LES DYNAMIQUES LINGUISTIQUES \\ DE LA SOCIÉTE ITALIENNE (1861-1980) : de la naissance de l'italien populaire à la diffusion des ethnicismes linguistiques}

\section{La situation sociolinguistique de l'Italie avant l'unité}

La situation sociolinguistique italienne, à l'époque de l'unité italienne (1861), était caractérisée par un fort déséquilibre entre les pôles linguistiques fondamentaux de l'italien et du dialecte; déséquilibre linguistique qui reproduisait le déséquilibre social existant entre classes hégémoniques et classes subalternes. L'italien, langue nationale officielle, était d'un usage très restreint, d'élite, limité à la couche dirigeante, essentiellement intellectuelle. D'autre part, l'usage de l'italien était presque complètement réservé à l'écrit même parmi les classes dominantes, qui, pour l'interaction orale, utilisaient selon lis situations ou une grande langue étrangère de culture (comme le français ou l'espagnol), ou le dialecte urbain du lieu de résidence. DE MAURO estime qu'à l'époque de l'unité l'italien n'était pas parlé par plus de $2,5 \%$ de la population 1 .

L'italien naît aux $X I I-X I I I^{e}$ siècles comme langue écrite (à l'origine surtout comme langue administrative, mercantile, de compte) surtout dans les nouvelles classes bourgeoises émergentes, en opposition avec une pratique orale, qui, d'après ce que nous en savons, devait rester dialectale. Cette scission nette entre l'usage oral et l'usage écrit caractérise l'histoire linguistique italienne jusqu'en plein XIX ${ }^{e}$ siècle. La pratique orale s'étendait presque complètement à l'intérieur du pôle dialectal qui, comme on peut le supposer non sans fondement d'après les indices disponibles, devait être notablement stratifié selon les classes sociales, et d'autre part énormément fragmenté selon les aires géographiques. Les mille et un dialectes locaux étaient traversés de stratifications sociales qui en réduisaient la diversité à mesure qu'on s'approchait du centre urbain et des classes supérieures. La langue populaire, c'est-à-dire la langue de l'ensemble des classes subalternes, était orientée vers des variétés sociolinguistiques moins amples, plus locales, plus dialectales, donc moins influencées par l'italien, et était exclusivement orale. Pour les rares usages écrits on utilisait naturellement l'italien, mais presque personne ne savait écrire. Normalement dans chaque communauté il y avait un "alphabète ", c'est-à-dire une personne en mesure de lire

1. De Malro, Storia, p. 43. Je considère que l'estimation est très optimiste, parce qu'elle comprend dans le nombre des locuteurs italiens les Toscans et les Romains ; il est douteux que ces derniers aient parlé l'italien mais bien plutôt une variété voisine de l'italien. 
et d'écrire pour la communauté. L'" alphabète "n'était pas toujours un représentant des classés hégémoniques (prêtre, avocat, pharmacien, administrateur) ; à l'intérieur même des classes populaires il y avait souvent quelqu'un en mesure de lire et d'écrire, que ce soit quelqu'un du pays ou un colporteur professionnel ou semiprofessionnel (comme les chanteurs ambulants ou les mendiants qui se procuraient gîte et couvert en lisant des livres et en racontant des histoires pendant les veillées d'hiver). On constate depuis le XVI ${ }^{e}$ siècle, à travers l'édition populaire et les feuilles volantes, une consommation populaire de récits, poèmes, chansons en italien : il est à peine besoin de rappeler la fortune des Reali di Francia, de Gerrino il Meschino, de la Genoveffa del Brabante, des poèmes de Dante et du Tasse, des chansons des chanteurs ambulants, toutes œuvres composées en un italien littéraire, très recherché, académique (au moins dans les intentions), dont on retrouve des traces dans les textes témoins d'italien populaire, notamment les plus anciens. Ces textes étaient diffusés dans une pratique communautaire : l'" alphabète " lisait à haute voix, assurant, selon l'heureuse formule d'Italo SORDI, un "alphabétisme " de groupe face à un analphabétisme individuel.

Les classes de marginaux (vagabonds, mendiants, colporteurs, malfrats) parlaient l'argot $*$, registre linguistique qui greffe, sur la grammaire et sur la phonétique de la langue ou du dialecte local, un lexique " argotique " commun et quelques traits morphosyntaxiques spécifiques, communs dans une large mesure à tous les argots italiens. Il est très probable que ceux qui parlaient en argot (spécialement dans les villes) parlaient aussi en plus de l'argot la langue italienne, comme le montrent les très nombreux italianismes - très spécialement les académismes - du lexique argotique. Elles connaissaient certainement l'italien, ces catégories de locuteurs argotiques qui faisaient profession de "vendre de la culture ", chanteurs ambulants (qui vendaient les feuilles volantes), colporteurs de livres et d'estampes, conteurs, forains, acteurs ambulants, marionnettistes et bateleurs. La fonction de médiation culturelle entre les couches hégémoniques et les couches subalternes et les contacts permanents entre les diverses classes sociales imposaient à ces groupes de marginaux la connaissance de toute la gamme des registres linguistiques de la société.

Nous pouvons supposer l'articulation sociolinguistique suivante qui vaut - dans

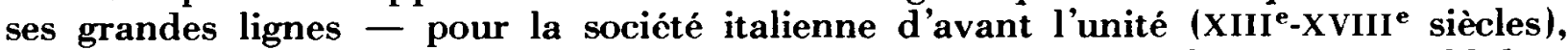
avec naturellement des différences profondes selon les zones et les époques : $1^{\circ}$ langue étrangère de culture, variable selon les zones (français, espagnol), d'usage oral et écrit dans les classes dominantes ; $2^{\circ}$ latin, d'usage écrit et partiellement oral aussi dans le clergé ; $3^{\circ}$ italien littéraire, d'usage écrit dans les classes dominantes, en particulier les intellectuels; $4^{\circ}$ koinè dialectale (régionale), fondée sur le dialecte d'un gros centre urbain, employée par les classes dominantes dans l'usage oral et aussi jusqu'à une certaine époque (variable selon les zones) dans l'usage écrit des chancelleries ou de la littérature (qu'on pense à la koinè lombardo-vénitienne des XII ${ }^{e}$ $X_{\text {III }}{ }^{e}$ siècles, à celle des chancelleries des cours de Milan et de Naples, ou au vénitien, langue officielle de la République de Venise) ; la koinè, à base dialectale, était modelée sur l'italien littéraire et sur le latin ; $5^{\circ}$ dialecte urbain civil, d'usage oral dans les classes dominantes et les couches moyennes des villes, et d'usage écrit littéraire, modelé sur l'italien littéraire directement ou par la médiation de la koinè dialectale ; $6^{\circ}$ dialecte urbain populaire, d'usage oral dans les classes populaires urbai-

* Argot correspondra ici à l'italien gergo et désignera non seulement l'« argot », proprement dit, langue des malfaiteurs, mais aussi les langues spéciales des marchands ambulants (N.d.T.). 
nes (ouvriers, artisans), fortement influencé par les ruralismes et les argotismes ; $7^{\circ}$ dialecte local civil, d'usage oral dans l'aristocratie et la classe d'administrateurs et d'artisans des petites villes, influencé par le dialecte urbain ; $8^{\circ}$ dialecte local rustique, d'usage oral chez les paysans ; c'est le registre linguistique le plus éloigné de l'italien ; $9^{\circ}$ Argot urbain, d'usage oral chez les marginaux urbains (forains, chanteurs ambulants, malfrats, etc.) notablement influencé par l'italien littéraire ; $10^{\circ}$ argot rural, d'usage oral chez les marginaux et les colporteurs des campagnes et des montagnes, qui se fondait sur les dialectes locaux.

Nous pouvons recueillir ceci en un tableau qui présente sous forme rigide une réalité linguistique qui devait être très mobile :

\section{TABleaU 1}

\begin{tabular}{|c|c|c|c|}
\hline VARIÉTÉ SOCIOLINGUISTIQUE & $\begin{array}{c}\text { SYSTÈME } \\
\text { LINGUISTIQUE }\end{array}$ & $\begin{array}{l}\text { MODALITÉ } \\
\text { D'USAGE }\end{array}$ & UTILISATEURS \\
\hline $\begin{array}{l}\text { 1. Langue étrangère } \\
\text { 2. Latin } \\
\text { 3. Italien littéraire } \\
\text { 4. Koiné dialectale } \\
\text { 5. Dialecte urbain civil } \\
\text { 6. Dialecte urbain populaire } \\
\text { 7. Dialecte local civil } \\
\text { 8. Dialecte local rustique } \\
\text { 9. Argot urbain } \\
\text { 10. Argot rural }\end{array}$ & $\begin{array}{l}\text { Langue étrangère } \\
\text { Latin } \\
\text { Italien } \\
\text { Dialecte } \\
- \\
- \\
- \\
- \\
\text { Argot }\end{array}$ & $\begin{array}{l}\text { oral, écrit } \\
\text { écrit } \\
\text { oral, écrit } \\
\text { oral }\end{array}$ & $\begin{array}{c}\text { classes hégémoniques } \\
\text { clergé } \\
\text { classes hégémoniques } \\
\text { classes hégémoniques } \\
\text { et couches moyennes } \\
\text { classes populaires } \\
\text { aristocratie et couches } \\
\text { moyennes } \\
\text { paysans } \\
\text { marginaux } \\
-\end{array}$ \\
\hline
\end{tabular}

\section{La naissance de l'italien populaire unitaire}

Au XIX ${ }^{e}$ siècle, avec la formation de l'État unitaire, l'institution du service militaire national obligatoire, la scolarisation, les progrès du mouvement politique et syndical et de la diffusion de la presse, commencent les grands processus socio-culturels dont l'intrication mènera à l'unification linguistique italienne et à la formation de l'italien populaire unitaire ; processus en apparence hétérogènes, en réalité mus et gouvernés par les mêmes causes socio-économiques : l'industrialisation, le développement du capitalisme et la formation de la classe ouvrière. L'Italie cesse d'être un pays rural et, au long d'un procès de " modernisation » encore en cours, elle commence à devenir un pays industriel.

Qu'est-ce que cela signifie sur le plan linguistique? L'économie paysanne, qui a eu son origine et son expression la plus accomplie à l'époque féodale, détermine la fragmentation de la société en de très nombreuses petites unités rurales isolées; dans une société paysanne, les besoins linguistiques sont limités aux contacts à l'intérieur de la communauté, alors que les contacts avec l'extérieur sont rares; la société paysanne produit, sur le plan linguistique, les dialectes; chaque bourg, chaque canton a sa propre langue locale, qui diffère toujours, peu ou prou, de la langue du bourg voisin. 
L'industrialisation a permis de dépasser l'isolement paysan et de concentrer les forces productives : moyens de production et force de travail, machines et d'hommes. Les usines naissent où les hommes se retrouvent par centaines, par milliers ; les cités industrielles naissent là où vivent et travaillent les ouvriers par dizaines et par centaines de milliers. L'industrialisation et l'urbanisation produisent le déplacement de grandes masses de population de la campagne vers la ville, du Mezzogiorno au triangle industriel (Milan-Turin-Gênes). Des millions d'hommes vont être arrachés à l'isolement de la vie paysanne, de la vie de bourg, pour être jetés dans l'agitation de la ville et de l'usine. Cela est à la source de nouveaux besoins linguistiques, qui provoquent la formation d'un nouvel instrument linguistique, l'italien populaire unitaire. Les mille dialectes locaux ne suffisent plus parce que dans les villes et dans les usines se retrouvent des hommes de cent lieux différents. Un instrument linguistique unifiant apparaît. Dans un premier temps il oscille entre l'italien et le dialecte urbain (à Milan le milanais, à Turin le turinois, etc.), ensuite l'italien prend le dessus car seul il peut garantir la formation d'un registre linguistique national, l'italien populaire, non I'italien littéraire de la bourgeoisie, mais l'italien comme peut et sait le parler le peuple, l'ensemble des classes subalternes ; italien populaire unitaire, parce que l'industrialisation surmonte les barrières des marchés locaux et adopte une structure nationale et, donc, parce qu'une langue nationale est la seule adéquate au niveau de développement de la société industrielle.

La classe ouvrière est le moteur de la formation du nouveau registre linguistique, qui est d'un usage essentiellement urbain. Dans les campagnes, les paysans continuent à parler le dialecte, sans ignorer pourtant l'italien. C'est dans les villes, dans les usines que l'italien populaire s'est formé et affirmé. L'italien populaire est témoin et expression d'un procès d'acculturation profondément dialectique : pendant que les classes hégémoniques, d'un côté, imposent l'italien aux masses, au moyen de l'école, du service militaire, de l'administration publique, ils imposent une langue unitaire répondant aux besoins de la société industrielle naissante, certes un type d'italien fortement appauvri, amputé de la richesse de la tradition littéraire, un "sous-produit » de l'italien littéraire, c'est-à-dire l'italien de l'école et de la bureaucratie ; d'un autre côté, les classes populaires s'emparent de cet italien, le modifient selon leurs propres traditions linguistiques, en font un instrument linguistique propre à des fins hégémoniques.

Une puissante impulsion à l'italianisation a été provoquée par la propagande politique et syndicale. Le développement et l'implantation des organisations syndicales et politiques, liés au procès d'industrialisation, entraînèrent une plus grande connaissance de l'italien, en particulier d'un italien académique, grandiloquent, rhétorique. La propagande des agitateurs socialistes était faite en langue italienne et de même les journaux et bulletins étaient rédigés en italien. L'activité politique et syndicale promut puissamment la diffusion de la lecture et l'alphabétisation. Les chants populaires à sujets politiques et syndicaux, les "chants sociaux », diffusés massivement au $X I X^{\circ}$ siècle, sont presque toujours en italien et continuent linguistiquement et musicalement la traduction des hymnes du Risorgimento ; cela aussi témoigne du dépassement de la société paysanne et $d u$ contact culturel auquel $j$ 'ai fait allusion plus haut. Aujourd hui encore l'activité politique et syndicale constitue un des véhicules principaux de l'italianisation linguistique.

La Première Guerre mondiale (1915-1918) et l'émigration donnèrent une impulsion décisive au processus de l'italianisation et de la formation de l'italien populaire unitaire. L'importance linguistique de la Grande Guerre est fondamentale et double : d'un côté elle met en ceuvre l'italianisation des grandes masses de paysans jusque-là exclus ou touchés marginalement par les processus linguistiques entraînés par l'indus- 
trialisation; de l'autre elle contraint paysans et ouvriers à se mesurer avec l'usage écrit actif de la langue et non plus seulement avec l'usage traditionnel oral.

L'italien populaire unitaire présente des éléments linguistiques de différenciation constitués par les traces du substrat dialectal, et des faits linguistiques unifiants dus à l'évolution interne. Il est préférable de distinguer entre les éléments linguistiques de différenciation et d'unification, plutôt qu'entre les "dialectalismes " et les faits d'évolution interne, dans la mesure où, s'il est vrai que les éléments de différenciation sont dus au substrat dialectal, tous les « dialectalismes " ne sont pas facteurs de différenciation. L'évolution interne de l'italien populaire est due en réalité, elle aussi, au contact linguistique, et nous y trouvons un lieu fondamental de contact avec les systèmes dialectaux, soit en un sens positif avec l'introduction des "dialectalismes", soit en un sens négatif, à travers l'introduction de traits d'hypercorrection, c'est-à-dire le choix de la forme qui s'éloigne le plus de la forme dialectale (même lorsque celle-ci coïncide avec la forme italienne littéraire). Dans tous les cas il est préférable de parler d'évolution interne et non d'interférence quand les apports externes sont unifiés et insérés de façon stable dans le système linguistique. Les éléments de différenciation sont surtout évidents au niveau phonétique-phonologique, tandis que les faits unifiants se manifestent en particulier au niveau morpho-syntaxique. L'italien populaire, pourtant, a, comme toute langue, son propre développement interne, dans la mesure où il se libère aussi bien de la base populaire que du modèle littéraire, développement qui suit les lignes directrices (typiques des langues populaires) de l'économie et de la régularité analogique.

\section{L'italien régional}

Depuis l'Unité, les mêmes processus qui ont déterminé dans la classe ouvrière la formation d'un italien populaire ont déterminé, dans d'autres couches sociales, la formation des registres linguistiques de l'italien régional et de l'italien bureaucratique. Paradoxalement, l'italien régional, issu d'un processus d'unification, représente une fragmentation de l'italien littéraire. Le paradoxe est pourtant seulement apparent : l'italien littéraire (standard) était unitaire seulement au niveau graphique, ayant eu un usage oral très limité ; cet usage oral était certainement différencié régionalement, mais de telles fragmentations n'étaient pas socialement pertinentes étant donné que cet usage était extrêmement réduit.

Les procès d'unification économique et politique ont diffusé la pratique orale de l'italien et ainsi la différenciation régionale, due aux diverses habitudes dialectales (actions du substrat) a surgi comme un phénomène important. Ayant été diffusé principalement au moyen de l'écrit, l'italien se différencie aussi régionalement par les diverses prononciations que l'ambiguïté de la graphie italienne a rendues possibles (graphie de $e, o, s, z, g n, g l, s c$, absence de marque graphique du redoublement phonosyntaxique, etc.). Par exemple, le -s- intervocalique qui se prononce comme une sourde ou une sonore suivant les mots (à la base de l'usage florentin), mais qui s'écrit toujours $s$ (ainsi casa devrait se prononcer ['ka:sa] mais rosa devrait se prononcer ['r s:za], tandis que dans l'Italie septentrionale on prononce tout en sonore ['ka:za] et ['ro:za], et que dans l'Italie centro-méridionale on prononce tout en sourde ['ka:sa] et ['ro:sa]).

L'italien régional se différencie de l'italien littéraire (langue de fantasme en ce qui regarde le parler, somme abstraite de règles) surtout au niveau phonétique ; les différences morpho-syntaxiques sont très légères alors que les différences lexicales sont plus importantes et qu'elles peuvent affleurer ainsi dans l'usage écrit : par exemple au mot toscan scodella " assiette creuse 》 correspond fondina dans le nord et piatto 
fondo ou piatto cupo dans le Sud ; à ciotola («bol 》) en Toscane correspond scodella dans le Nord, et tazza dans le Sud; etc. L'italien régional reflète l'usage local de l'italien littéraire, usage accepté comme correct et enseigné à l'école ${ }^{2}$. En pratique l'italien régional représente pour la majeure partie de la population la norme, ceux qui connaissent effectivement la norme littéraire standard et l'appliquent étant très rares (en particulier pour ce qui regarde la prononciation). L'usage de l'italien régional est diffusé dans les classes dominantes (haute et moyenne bourgeoisie, intellectuels). On peut distinguer un niveau plus surveillé employé à l'écrit et dans la conversation formelle, et un niveau bas, l'italien de conversation (colloquial), employé de façon non formelle dans l'usage parlé, registre proche de l'italien populaire.

\section{L'italien bureaucratique}

L'italien bureaucratique est issu de la centralisation administrative, il vient des ministères, des tribunaux, des offices publics, des diverses branches de l'administration étatique. Il est né de l'écrit, sur le modèle du langage juridique, dont il voudrait avoir la précision et l'univocité, mais dont il ne parvient qu'à calquer la complexité et la bizarrerie. Néanmoins, comme l'a écrit Pietro Cítati 3 , "il recherche, plus ou moins consciemment, un idéal esthétique. Avec les moyens expressifs d'un analphabète, il s'efforce de répéter la dignité du cursus latin ". Langue "née et cultivée amoureusement dans les antichambres des ministres, dans les études des avocats (...), capable d'orner les plaidoiries de nos hommes politiques " (P. CITATI), c'est la langue des circulaires administratives, des rapports de police, des actes judiciaires, des discours officiels. C'est une langue créée par la petite bourgeoisie administrative d'origine centro-méridionale qui, grâce au transfert à Rome de la capitale (1870), a massivement occupé l'administration publique et s'est identifiée à la bureaucratie, aux tribunaux, à la politique. De là l'italien bureaucratique s'est étendu aux journaux, et à l'école où domine ce qu'on appelle l' "italien scolaire ", langue imitée des circulaires de la hiérarchie, langue qui induit les enseignants à corriger faccia ("visage») en volto, passare le vacanze ("passer les vacances $"$ ) en trascorrere le vacanze, etc.

L'italien bureaucratique est l'antilangue d'Italo CaLvino ${ }^{4}$, caractérisée du point de vue du style par la " terreur sémantique ", " c'est-à-dire la fuite devant tout vocable qui aurait un signifié par lui-même (...) l'italien de celui qui ne sait pas dire "j'ai fait " (ho fatto), mais se doit de dire " j'ai effectué " (ho effettuato) ". A l'origine il y a le refus du langage courant, du langage parlé qu'on juge imprécis et peu sûr, au profit d'un langage qui dans ses intentions devait être plus précis, objectif et en quelque sorte "formalisé » de façon stable suivant le modèle du langage juridique.

L'italien bureaucratique répugne à une quelconque "contamination " par la forme linguistique de l'usage parlé ; le principe de l'hypercorrection, qui consiste à choisir entre deux formes concurrentes celle qui est la plus éloignée de l'usage parlé, est appliqué avec rigueur : effettuare ( effectuer "), eseguire ( exécuter ") au lieu de fare (" faire »), il quale ( lequel ») au lieu de che ("qui »), etc. On rencontre fré-

2. La prononciation régionale est enseignée - dans la majeure partie des cas - inconsciemment, l'école italienne n'ayant aucune tradition d'enseignement de l'orthoépie. Les enseignants, individuellement, ignorent la norme phonétique standard.

3. Pietro Cítati, L'italiano sta diventando un gergo furbesco, dans Il Giorno du 20 janvier 1965, repris dans Parlangeli, Questione, p. 119.

4. Italo Calvino, Per ora sommersi dall'antilingua, dans Il Giorno du 3 février 1965, repris dans Parlangeli, Questione, p. 173 ; Colombo, p. 140. 
quemment des formules stéréotypées cristallisées, à enchâsser dans des énoncés " modulaires", suivant un style de formulaires bureaucratiques, dans lesquels une bonne part est donnée aux déictiques signifiant soussigné ou susdit : sottoscritto, predetto, suddetto, infrascritto, sullodato, etc.

À l'origine usage administratif écrit, l'italien bureaucratique est passé aussi à l'usage oral suivant le principe " parler comme un livre », c'est-à-dire " parler comme on écrit ", principe absurde diffusé par l'école. Il est fréquent d'entendre parler l'antilangue de CALviNo, spécialement (mais pas seulement !) en situation formelle, particulièrement de la part de la petite bourgeoisie du tertiaire. Cet usage est à la base d'une plaisanterie sur deux gendarmes recevant l'ordre "de se porter sur la place de la cathédrale et, de là, de se pousser dans la rue Dante " ce qui en italien non-bureaucratique veut dire " aller sur la place de la cathédrale et de là continuer vers la rue Dante ». L'usage de ce registre linguistique s'est naturellement accru et consolidé sous le fascisme.

\section{Crises et transformations des dialectes}

Le procès qui a abouti à la naissance et à la diffusion de l'italien populaire unitaire a déterminé aussi la réduction simultanée de l'usage des dialectes et leur rapprochement progressif de l'italien. L'industrialisation du pays a produit une réduction massive des locuteurs dialectaux des campagnes, à la suite de la réduction progressive du nombre des paysans; ce phénomène est sensible surtout dans la seconde après-guerre (1945-1980), après une période de pause entre les deux guerres (19181940), favorisée par la politique ruraliste du fascisme. Les dialectes, en plus du fait qu'ils ont vu se réduire sensiblement le nombre de leurs locuteurs, ont subi un processus accéléré d'italianisation : en milanais par exemple, pour dire sicuro (" sûr 》) on dit sicüür [si'ky:r], forme imitée de l'italien par rapport à la forme d'autrefois sigüïr [si'gy:r], encore présente dans l'usage plus populaire, forme qui, de son côté, était l'italianisation par restauration du $-r$ - final (dans le dialecte celui-ci tombe), d'une forme antérieure sigii [si'gy] attestée encore aujourd'hui à la campagne. Pareillement, des éléments linguistiques non présents aussi en italien ont disparu, comme quelques phonèms ou les oppositions de longueurs vocaliques : dans les dialectes de la Vénétie par exemple, $\theta$ est remplacé par [s]; en milanais, la distinction entre voyelles longues et brèves est en régression. On observe un processus de restauration sur la base du modèle italien, aussi bien au niveau phonétique qu'au niveau lexical : en milanais, lac' ['lats] " lait " (italien latte) est remplacé par lat ['lat]; en bolognais arguèerd [ar'gw $:$ :rd] "regarde " (italien riguardo) est remplacé par riguèerd [ri'gwe:rd]; dans les dialectes de la Vénétie, pirón [pi'rơ] "fourchette" (italien forchetta) est remplacé par forchéta [for'keta], et ainsi de suite.

En vérité l'italianisation des dialectes est un phénomène très ancien, datant de l'époque même de la formation de la langue italienne, qui a toujours influé sur eux en les remodelant. Qu'on pense au cas du milanais qui, au moins depuis le XIII' cle, a progressivement éliminé les traits les plus dialectaux pour se rapprocher du modèle italien avec l'élimination, par exemple, de la métaphonie, très vivante au $\mathrm{XII}^{\mathbf{e}}$ siècle; avec la restitution du $-l$ - intervocalique, passé à -r- dans le dialecte (encore au début du XVII ${ }^{e}$ siècle on trouve Miran "Milano ", à côté de Milan) ; avec l'élimination du son $s g\left[{ }_{j}\right]$, inconnu de l'italien, remplacé par $g^{\prime}\left[d_{j}\right]$. L'italianisation du dialecte est très profonde dans les zones où se sont formées les koinè dialectales 
régionales, zones que John TRUMPER 5 a nommées macrodiglossies (Lombardie et Vénétie, Piémont Centre et Nord, Campanie, Sicile), où l'importance de l'usage, même public, de la koinè dialectale est encore aujourd'hui considérable et où on trouve des superpositions de fonctions entre dialectes et italien. Dans la situation opposée, où il n'y a pas eu de formation d'une koinè dialectale, on a alors une microdiglossie (Piémont méridional, Emilie, Romagne, Marches, Calabre) : les fonctions de l'italien et du dialecte sont bien séparées ; le dialecte (patois), destiné uniquement aux usages locaux et familiaux, est moins influencé par l'italien et se maintient dans une forme très figée. Alberto M. MIONI 6 souligne que la "pureté » de ce dialecte est indice d'une faible vitalité, d'un danger sérieux d'extinction et de remplacement par l'italien, alors que l'italianisation rend plus stables les dialectes en situation de macrodiglossie : la capacité d'adaptation exclut le danger immédiat de remplacement par l'italien : le dialecte subit plutôt une transformation progressive sur le modèle italien.

Quelles lignes suit le processus d'italianisation des dialectes ? En réalité nous n'avons pas affaire simplement au remplacement des traits linguistiques non présents en italien : comment expliquer alors la persistance des sons $\ddot{o}[\phi], \ddot{u}[y]$ dans les dialectes lombards, ou la perte récente de $z$ [ts] en milanais (son présent en italien !) remplacé par $s$ [s] ; mais surtout comment expliquer la vitalité et la force d'expansion de ces traits dialectaux qui, au lieu de disparaître en tant que différents de l'italien, pénètrent tout à fait dans l'italien populaire (qu'on pense à la prononciation lombarde tüto [ty:to] « tout " (italien tutto), ragasso [ra'gasso] "garçon » (italien ragazzo) ? Nous voyons donc que quelques différences spécifiques entre dialecte et italien ont été maintenues et consolidées. L'italianisation des dialectes se réalise non pas au moyen d'une substitution phonétique complète mais par la construction d'un parallélisme phonétique analogique (si possible biunivoque) par lequel, d'une forme italienne, on peut facilement passer à une forme dialectale et vice versa. En prenant par exemple le milanais, nous voyons que le maintien de $\ddot{u}[y]$ est possible à l'intérieur d'un réseau régulier de correspondance où, à $u[u]$ italien, correspond toujours $\ddot{i}$ en dialecte (muro ['mu:ro [müür ['my:r] " mur »), comme à $-t$ - intervocalique italien correspond toujours $-d$ - dans le dialecte (fratello [fra't tello] fradel [fra'dgl] «frère "). Ainsi est non seulement possible, dans la ligne du principe de " rotation», mais encore nécessaire l'élimination de ces changements phonétiques trop radicaux ou irréguliers qui rendent impossible la création d'un système actif de quasi "translittération ». Évidemment, pour appliquer un semblable mécanisme il faut une homogénéisation lexicale; pour passer activement de la phonétique italienne à la phonétique dialectale, il faut que les bases lexicales soient communes : nous avons ainsi l'élimination progressive des types lexicaux dialectaux non conformes à l'italien (sauf le maintien de quelques formes très caractéristiques comme signe de l'altérité dialectale) : par exemple en milanais le terme erbiu: [er'bjū:] " petit pois " est tombé en désuétude, remplacé par pisei [pi'zєj], transposition phonétique de l'italien piselli.

Le processus de remodelage des dialectes sur l'italien s'est grandement accentué après la seconde guerre avec la diffusion capillaire des grands moyens de communication de masse (radio, télévision, cinéma, presse) qui contribuent de manière décisive au processus d'italianisation.

Le passage des dialectes à l'italien (substitution) et l'italianisation des dialectes (transformation), la naissance et la diffusion de l'italien populaire unitaire, des ita-

5. Voir Trumper, Ricostruzione.

6. Voir Mioni-Arnuzzo Lanszweert, p. 94 ; Mioni, Situazione, p. 109. 
liens régionaux, de l'italien bureaucratique s'accompagnent de la modification de l'italien littéraire standard.

Le processus linguistique le plus important auquel nous assistons est précisément la modification progressive de l'italien littéraire sous la poussée de l'italien populaire, qui tend à imposer son propre usage linguistique et à se proposer comme future langue standard.

L'italien populaire a comme point de référence l'italien de la norme écrite, langue vers laquelle tend, comme vers un modèle, le locuteur, et a comme point de départ les vieux usages dialectaux et argotiques; mais l'italien populaire est en train de modifier progressivement la vieille norme écrite. Le rapport complexe entre italien populaire, italien littéraire et dialecte semble se résoudre dans une interaction toujours plus étroite entre les deux premiers registres linguistiques, avec une marginalisation progressive du registre dialectal.

\section{La diffusion des ethnicismes linguistiques}

L'expansion de l'italien populaire, liée à la croissance du poids politique des couches populaires, spécialement de la classe ouvrière, a provoqué des phénomènes de réaction sur le plan linguistique et culturel, c'est l'apparition des ethnicismes linguistiques.

Actuellement nous assistons à de nombreuses tentatives de réhabilitation du dialecte, langue " authentiquement populaire " et " opprimée "; elles sont dirigées contre l'italien, langue de l'oppression bourgeoise, et s'apparentent aux opérations de relance des minorités ethnico-linguistiques. Ce revival ne naît pas dans les couches populaires, il n'est pas proposé par les utilisateurs effectifs du dialecte, mais il est l'expression de la récupération nostalgique des traditions pré-bourgeoises de la part des intellectuels petits-bourgeois, spécialement de province, liée à des réalités locales objectivement marginalisées par les processus de centralisation.

À la fin des années soixante, on assiste à une explosion des ethnicismes linguistiques. Les premiers mouvements de revendication des minorités linguistiques ont lieu dans les zones périphériques, dans ces populations qui par leur différence culturelle (vraie ou présumée) ou par une tradition autonomiste antérieure peuvent se reconnaître une quelconque identité " nationale " (Sardaigne, vallées piémontaises de langue occitane, Frioul). Témoignent de cette première phase, liée au concept de "nation" du siècle dernier (et de "peuple-nation"), les travaux de Sergio SALVI. Dans les années 70 , on passe à une seconde phase de plus amples revendications "localistes" (dont sont témoins principalement les travaux du sociologue catholique Ulderico BERNARDI) : l'autonomie linguistique et culturelle est revendiquée pour chaque "communauté ", donc pour tout dialecte affronté au débordement d'un italien de masse, destructeur de toutes les particularités locales. En Italie, un processus d'unification économique, culturelle, civile et linguistique est en train de se développer avec une accélération progressive. Ce processus provoque deux réactions diverses : l'une tournée vers l'avenir considère la classe comme contradiction principale ; l'autre, tournée vers le passé, l'ethnie.

Il y a donc une réponse qui ne passe pas par les oppositions de classes, mais par l'agrégation interclassiste autour de valeurs formelles (et mal fondées) telles que race, langue, nation, ethnie. La lutte se développerait entre une langue et une ethnie, en bloc (exploités et exploiteurs ensemble), contre une autre langue et une autre ethnie, elles aussi prises en bloc.

La situation de gêne aigüe vécue par les couches moyennes, et en particulier par celles des aires marginales, se manifeste dans une tentative de récupération du passé, 
des valeurs culturelles traditionnelles telles que le dialecte, la "communauté », le folklore, les traditions héréditaires, toutes choses bousculées par la civilisation industrielle, vue comme un monstre omnivore qui unifie tout et détruit tout.

Les valeurs à récupérer ne sont pas les valeurs traditionnelles propres aux couches moyennes, mais les valeurs paysannes, c'est-à-dire celles d'une autre classe, désormais dépassée par le développement des rapports sociaux, qui est adoptée "idéologiquement " comme symbole d'une société à restaurer contre la société actuelle.

\section{La situation sociolinguistique italienne (1980)}

Au terme de ce panorama que nous avons esquissé nous pouvons essayer de dessiner le tableau de la situation sociolinguistique actuelle (1980), de la confronter avec celle de départ proposée au $1^{\text {er }}$ paragraphe, en avertissant que celle-ci, comme cellelà, n'est que l'extrême simplification et la rigidification d'une réalité très mobile et complexe. Les pôles linguistiques fondamentaux de l'italien normatif (italien littéraire ou régional), du dialecte et de l'argot ne sont pas assumés dans leur intégrité pleine et abstraite, mais les locuteurs actualisent ces systèmes dans toute une série de variétés, c'est-à-dire de niveaux de réalisation et d'intégration dépendant des capacités linguistiques du locuteur, du contexte, de la nécessité sociale et des besoins expressifs.

La réalité sociolinguistique italienne peut être segmentée, dans ses lignes essentielles, en seize variétés. Le locuteur a la compétence active de certaines variétés, il n'a que la compétence passive d'autres, et il n'en a aucune d'autres encore (c'est le cas, pour une large partie de la population, des variétés argotiques). Les variétés isolées ne doivent absolument pas être entendues de façon rigide : ce sont des abstractions utiles à la description de l'articulation de l'usage linguistique qui, concrètement, se situe au niveau de ces variétés comme aux niveaux intermédiaires; les passages d'une variété à l'autre en cours de conversation (dus principalement à des motivations expressives) ne sont pas rares. Le choix de la variété est déterminé par l'interlocuteur ; quand il est impossible d'utiliser une variété commune, le locuteur fait usage de la variété la plus proche de celle de l'allocutaire : les interlocuteurs ont une compétence active de leur propre variété et une compétence passive de la variété de l'allocutaire. Cette situation se vérifie très fréquemment à l'occasion des interactions entre locuteurs de groupes sociaux différents ou entre locuteurs d'aires géographiques différentes.

1) Italien anglicisé (ou anglo-italien) : variété autant orale qu'écrite, caractérisée par des phénomènes $d$ 'interférences avec l'anglais et par une forte présence, dans le lexique, d'anglicismes et de termes du lexique européen savant. Utilisé par la haute bourgeoisie qui a des contacts internationaux, et par les managers, il tend à se diffuser chez les employés de l'industrie et chez les journalistes. Signe de l'intégration progressive de l'italien dans le système culturel mondial du néocapitalisme, il peut représenter une des futures lignes de développement de la langue ${ }^{7}$.

2) Italien littéraire (standard) : langue de l'usage littéraire codifiée par la grammaire et les manuels de prononciation, elle est presque complètement limitée à l'usage écrit et elle est en train, dans ce domaine, de perdre du terrain au profit de l'italien régional et de l'italien bureaucratique. Employée par la haute bourgeoisie et par les intellectuels, elle représente la norme linguistique de droit.

3) Italien régional : il représente la norme linguistique (standard) de fait. Comme nous l'avons vu au paragraphe 3 , c'est la réalisation concrète de l'italien littéraire

7. Le sociologue Francesco Alberoni a récemment proposé d'abandonner l'usage de l'italien au profit de l'anglais. 
dans l'usage oral formel et désormais dans une large mesure aussi dans l'usage écrit. Il présente des variantes locales (généralement régionales) surtout au niveau phonétique et lexical, moins au niveau morpho-syntaxique. Les variantes permettent en quelque sorte une intercompréhension sûre et donnent en outre la possibilité (surtout dans l'usage oral, beaucoup moins à l'écrit) d'identifier l'origine géographique de l'interlocuteur. Il est employé par la bourgeoisie et les intellectuels.

4) Italien " colloquial " c'est la réalisation orale courante et informelle de l'italien régional. Les localismes phonétiques et lexicaux et surtout les déviances syntaxiques par rapport à la norme littéraire y sont accentués ; de ce point de vue, l'italien " de conversation " se rapproche de l'italien populaire. L'usage de cette variété est présent dans de larges couches qui vont de la bourgeoisie aux couches moyennes.

5) Italien bureaucratique : c'est la variété, orale également mais principalement écrite, illustrée au paragraphe 4. Employée par les couches moyennes du tertiaire (surtout de la fonction publique), c'est le modèle de l'italien normalement proposé par les enseignants à l'école.

6) Italien populaire (unitaire) : variété illustrée au paragraphe 2, elle est diffusée dans les classes populaires (spécialement dans la classe ouvrière). Comme les trois variétés précédentes (italien régional, "de conversation ", bureaucratique) elle présente des variations régionales, phonétiques et lexicales, mais surtout une diversité syntaxique notable par rapport à la norme. En raison de la tendance à l'économie, d'une part, et de l'incertitude continuelle en ce qui concerne la norme d'autre part, l'italien populaire est sujet à tous les types d influence et de pression : de la part de l'italien littéraire et académique, senti comme plus « noble » et plus proche d'un italien qu'on ne possède pas bien mais auquel on tient ; de la part des langages spécialisés : bureaucratiques, politiques, syndicaux, techniques, publicitaires, radiotélévisuels; de la part du dialecte du locuteur qui affleure continuellement et n'est pas toujours contrôlé ni contrôlable ; de la part des dialectes divers avec lesquels on entre en contact, des argots, des jargons, des mots étrangers. D'usage principalement oral, on en a étudié surtout des performances écrites (lettres et journaux d'illettrés), qui représentent un usage quantitativement marginal mais anthropologiquement assez pertinent.

7) Italien dialectal : c'est la réalisation de l'italien populaire de la part du sujet fortement dialectophone n'ayant que peu de familiarité avec l'usage de la langue italienne. L'interférence dialectale est très forte. C'est une variété principalement orale, utilisée par les classes populaires.

8) Italien-dialecte : variété caractérisée par le passage continuel de l'expression italienne à l'expression dialectale, par la production continuelle d'énoncés en « langue mixte ", liée à des motivations stylistiques au sens large et aussi à l'incapacité effective de maintenir le discours en italien. Il est présent surtout dans les situations de macrodiglossie (paragraphe 5), dans l'usage oral des classes populaires.

9) Dialecte italianisé : variété exclusivement orale, propre aux classes populaires des grandes villes, il représente une très forte italianisation du dialecte, ou mieux un travestissement dialectal de l'italien au niveau phonétique (opéré selon le réseau de parallélismes phonétiques dont il a été question au paragraphe 5) : qu'on considère par exemple les formes utumòbil [utu'mo:bil] « automobile » (italien automobile), separasiū [separa'sjũ] " séparation » (italien separazione), dirigêt [diri'gẽ:t] « dirigeant » (italien dirigente) du milanais italianisé.

10) Koinè dialectale (régionale): variété linguistique présente seulement dans les situations de macrodiglossie (paragraphe 5), à l'origine de la diffusion, sur une aire 
vaste, de l'usage dialectal d'un grand centre, avec éventuellement quelques légères modifications. De fortes interférences avec l'italien y sont présentes, qui sont aussi très anciennes (paragraphe 5). Le cas le plus évident est présenté par le dialecte commun de Vénétie ayant pour base le vénitien. L'usage est oral (l'écrit est limité au style littéraire, employé par les poètes dialectaux), avec des variations notables selon les zones en ce qui concerne les fonctions et le milieu sociologique : cela va de la bourgeoisie (Vénétie) aux couches moyennes et aux couches populaires.

11) Dialecte urbain (provincial) : dialecte des centres urbains grands ou moyens et des territoires qui en dépendent, c'est le plus italianisé des dialectes locaux. Il est d'un usage oral (l'écrit est limité au style littéraire), diffusé - avec des variations selon les zones - dans les couches moyennes et les classes populaires. Il existe aussi un usage aristocratique restreint du dialecte urbain (familles nobles, propriétaires terriens), orienté vers le style littéraire archaïque, en opposition avec la pression exercée par l'italien et avec le dialecte des classes populaires et des immigrés.

12) Dialecte local civil : dialecte des petits centres, influencé par l'italien et par le dialecte urbain, il a un emploi oral (écrit seulement dans le style littéraire) dans les couches moyennes des petites villes.

13) Dialecte local rustique : c'est la forme du dialecte la plus éloignée de l'italien, plus conservatrice, exclusivement orale, utilisée par les paysans.

14) Argot italien (régional) : variété régionale de l'argot italien commun, d'usage exclusivement oral, principalement chez les prisonniers, les délinquants, les malfrats, les forains.

15) Argot dialectal urbàin : argot basé sur le dialecte urbain, d'usage oral chez les classes de marginaux des villes.

16) Argot dialectal rustique : argot basé sur les dialectes locaux, d'usage oral chez les colporteurs de la campagne et de la montagne.

Nous pouvons rassembler en un tableau les lignes fondamentales de cet exposé (tableau 2).

Le schéma proposé représente une abstraction maximum, qui couvre des situations particulières (géographiques et sociales) très diverses aussi. On n'a pas mis en évidence la différence fondamentale entre l'usage des zones sans immigration, orienté vers des dialectes homogènes, et l'usage des zones d'immigration, surtout l'aire du triangle industriel (Turin-Milan-Gênes), où se superposent les traditions dialectales très différentes apportées par les immigrés. En outre il faut tenir compte des styles (formel, informel, littéraire, etc.), qui en partie coïncident avec les variétés (l’italien " de conversation " peut être considéré comme le style informel de l'italien régional), en partie non : le dialecte urbain, par exemple, a un style formel (le plus élevé), un style informel (le plus populaire), et un style littéraire (archaïsant, utilisé par les poètes dialectaux). Le choix qui nous conduit à considérer l'italien "de conversation " comme une variété et non comme un simple style est lié à une valorisation importante d'un point de vue sociolinguistique. En l'absence de recherches empiriques, le critère de jugement subjectif formulé sur la base de l'expérience et de la sensibilité du linguiste prévaut.

Les seize variétés énumérées ne sont pas toutes sur le même plan. Un choix aussi large se propose de rendre compte d'un usage social de la langue très riche et différencié. Néanmoins, certaines variétés doivent être considérées en premier rang : ce sont les registres sociolinguistiques autour desquels s'agrègent les variétés linguistiques secondaires. Les registres sociolinguistiques sont directement produits par une classe sociale et peuvent s'articuler en plusieurs variétés. 
TABleau 2. Les variétés de 2 à 13 (système italien-dialecte) et de 14 à 16 (système argotique) sont ordonnées d'un maximum d'unité à un maximum de diversité ; chaque variété successive y implique les localismes de la variété précédente.

\begin{tabular}{|c|c|c|c|}
\hline VARIÉTÉ SOCIOLINGUISTIQUE & $\begin{array}{c}\text { SYSTĖME } \\
\text { LINGUISTIQUE }\end{array}$ & $\begin{array}{c}\text { MODALITÉ } \\
\text { D'USAGE }\end{array}$ & UTILISATEURS \\
\hline 1. Italien anglicisé & Italien & oral, écrit & bourgeoisie \\
\hline 2. Italien littéraire standard & - & écrit & - \\
\hline 3. Italien régional & - & oral, écrit & - \\
\hline 4. Italien « colloquial » & - & oral & $\begin{array}{l}\text { bourgeoisie, couches } \\
\text { movennes }\end{array}$ \\
\hline 5. Italien bureaucratique & - & oral, écrit & couches moyennes \\
\hline 6. Italien populaire (unitaire) & - & oral & $\begin{array}{l}\text { classes populaires, } \\
\text { couches moyennes }\end{array}$ \\
\hline 7. Italien dialectal & - & - & classes populaires \\
\hline 8. Italien-dialecte & Mixte & - & - \\
\hline 9. Dialecte italianisé & Dialecte & - & - \\
\hline 10. Koinè dialectale (régionale) & - & - & $\begin{array}{l}\text { classes populaires, } \\
\text { couches moyennes }\end{array}$ \\
\hline 11. Dialecte urbain (provincial) & - & - & - \\
\hline 12. Dialecte local civil & - & - & - \\
\hline 13. Dialecte local rustique & - & - & paysans \\
\hline 14. Argot italien (régional) & Argot & - & $\begin{array}{l}\text { classes de margi- } \\
\text { naux }\end{array}$ \\
\hline 15. Argot dialectal u & - & - & - \\
\hline 16. Argot dialectal rustique & - & - & - \\
\hline
\end{tabular}

Nous pouvons donc reformuler le tableau ci-dessus, considérant les seuls registres sociolinguistiques (tableau 3 , p. suiv.).

La détermination des répertoires linguistiques à la disposition des locuteurs des différentes classes sociales n'est pas facile. Une première indication sommaire est offerte par le tableau 2. On gardera présent à l'esprit qu'une partie de plus en plus importante des locuteurs italiens, spécialement dans les grandes cités, ignore le dialecte, possède l'italien comme unique instrument linguistique et constitue pourtant la strate linguistique la plus pauvre de la société. Le schéma proposé pour illustrer la situation sociolinguistique italienne est malheureusement statique. Pour offrir un élément d'analyse dynamique du passage du dialecte à l'italien on peut proposer quelques exemples, très simplifiés, des rapports linguistiques entre les différentes générations, en adaptant le schéma de MIONI 8 :

A) Zone urbaine hautement industrialisée :

Grands-parents \{ compétence active du dialecte

$\left\{\begin{array}{l}\text { compétence active de l'italien } \\ \text { compétence active du dialecte }\end{array}\right\}$ dialecte

Parents

$\left\{\begin{array}{l}\text { compétence active de l'italien } \\ \text { compétence passive du dialecte }\end{array}\right\}$ italien

Enfants

8. Voir Mioni-Arnuzzo Lanszwhert, p. 97. 
Tableau 3

\begin{tabular}{|c|c|c|c|}
\hline $\begin{array}{c}\text { REgISTRE } \\
\text { SOCIOLINGUISTIQUE }\end{array}$ & $\begin{array}{c}\text { VARIÉTÉ } \\
\text { SOCIOLINGUISTIQUE }\end{array}$ & $\begin{array}{c}\text { SYSTĖME } \\
\text { LINGUISTIQUE }\end{array}$ & $\begin{array}{l}\text { Classe Sociale } \\
\text { Ȧ L'ORIGINE }\end{array}$ \\
\hline Italien standard & $\begin{array}{l}\text { 1. Italien anglicisé } \\
\text { 2. Italien littéraire } \\
\text { 3. Italien régional } \\
\text { 4. Italien " colloquial " }\end{array}$ & Italien & bourgeoisie \\
\hline Italien bureaucratique & $\begin{array}{l}\text { 5. Italien bureau- } \\
\text { cratique }\end{array}$ & Italien & $\begin{array}{l}\text { petite bourgeoisie du } \\
\text { tertiaire }\end{array}$ \\
\hline Italien populaire unitaire & $\begin{array}{l}\text { 6. Italien populaire } \\
\text { 7. Italien dialectal } \\
\text { 8. Italien-dialecte } \\
\text { 9. Dialecte italianisé }\end{array}$ & $\begin{array}{c}\text { Italien } \\
\text { et dialecte }\end{array}$ & classe ouvrière \\
\hline Dialecte urbain & $\begin{array}{l}\text { 10. Koinè dialectale } \\
\text { 11. Dialecte urbain } \\
\text { 12. Dialecte local civil }\end{array}$ & Dialecte & $\begin{array}{l}\text { artisans et couches } \\
\text { moyennes pré-indus- } \\
\text { trielles }\end{array}$ \\
\hline Dialecte rustique & $\begin{array}{l}\text { 13. Dialecte local rus- } \\
\text { tique }\end{array}$ & Dialecte & paysans \\
\hline Argot & $\begin{array}{l}\text { 14. Argot italien } \\
\text { 15. Argot dialectal } \\
\text { urbain } \\
\text { 16. Argot dialectal rural }\end{array}$ & Argot & classes de marginaux \\
\hline
\end{tabular}

B) Centres moyens et petits et aires avec macrodiglossie :

$\left.\begin{array}{lll}\text { Grands-parents } & \begin{array}{l}\text { compétence active du dialecte } \\ \text { compétence passive de l'italien }\end{array} \\ \text { Parents } & \begin{array}{l}\text { compétence active du dialecte } \\ \text { compétence active de l'italien }\end{array} \\ \text { Enfants } & \begin{array}{l}\text { compétence active du dialecte } \\ \text { compétence active de l'italien }\end{array}\end{array}\right)$ dialecte $4 \uparrow$

C) Zones rurales :

$\begin{array}{ll}\text { Grands-parents } & \begin{array}{l}\text { compétence active du dialecte } \\ \text { compétence passive de l'italien }\end{array} \\ \text { Parents } & \begin{array}{l}\text { compétence active du dialecte } \\ \text { compétence active ou passive } \\ \text { de l'italien }\end{array} \\ \text { Enfants } & \begin{array}{l}\text { compétence active du dialecte } \\ \text { compétence active de l'italien }\end{array}\end{array} \quad$ dialecte

Dans les zones hautement industrialisées, l'italien s'impose aussi dans les relations verbales familières entre parents et enfants. Dans les zones intermédiaires, nous avons la situation « déséquilibrée " caractéristique (jusqu'à la dernière génération, 
typique aussi dans les zones industrielles) : les parents (et grands-parents) s'adressent aux enfants en dialecte, et ceux-ci répondent en italien. Dans les zones rurales le dialecte est encore le moyen de communication exclusif en famille, et l'italien est limité aux relations extérieures.

(mai 1980)

(Traduit de l'italien par Daniel BAGGIONI, Centre universitaire de l'Océan Indien.) 


\section{NOTES BIBLIOGRAPHIQUES}

Pour toute la matière traitée je renvoie à l'ouvre capitale de DE MAUro, Storia. Sur les rapports langue, culture, société, les observations éparses mais non gratuites de GraMSCI (sur ses positions linguistiques se rapporter à Lo Piparo ; Carrannante ; Rosiello, Gramscil sont très importantes. Pour les rapports entre langue et classes sociales je renvoie à SANGA, Principii ; Sanga, Dialetto ; Marcellesi-Ponzio ; pour les aspects politiques des dynamiques linguistiques, je renvoie à FISHMAN ; CARDona. Dans les transcriptions phonétiques, j'emploie le système simplifié basé sur la graphie italienne proposé par la Rivista italiana di dialettologia (voir SANGA, Trascrizione) en la faisant suivre de la transcription suivant le système A.P.I. entre crochets.

§ 1. - L'histoire de la langue italienne est magistralement traitée dans Migliorini, Storia ; voire en outre Devoto, Profilo ; Devoto, Linguaggio ; DE Mauro, Storia ; Stussi ; Migliorini-Baldelli ; Devoto-Altieri.

L'ouuvre la plus récente et la plus complète sur la "question de la langue » est Vital.E, Questione; on verra en outre MIgliorini, Questione; SOZZI. Pour le XIX ${ }^{\mathrm{e}}$ siècle on verra Áscoli, Questione. Pour le Xxe, Parlangeli, Questione.

Pour les dialectes on verra les introductions de Taglavini, Origini ; Cortelazzo, Avviamento ; les descriptions de Pellegrini, Carta ; Rohlfs, Studi ; les grammaires historiques de RoHlFs, Grammatica ; TEKAvČIĆ. En outre. on se reportera aux monographies en cours de publication dans la collection de Contelazzo, Profilo, et aux atlas linguistiques : italien (AIS, ALI) ; frioulan (ASLEF) ; corse (ALEIC), sarde (TERRACINI-FranceschI) ; toscan (ALT) ; méditerranéen (ALM) ; européen (ALE).

Comme répertoires bibliographiques assez complets, il y a Hall, Bibliografia ; Hall, Supplemento ; Parlangeli, Saggio ; Parlangeli, Bibliografia ; Arnuzzo-Marcato : BLI : MulJaČıć, Introduzione (bibliographie largement commentée) ; HALL, Bibliografia essenziale ; pour la période 1965-1975, les excellentes revues bibliographiques-critiques rassemblées dans SLI Dieci anni sont des synthèses très utiles ; on verra en outre le large fichier bibliographique rassemblé dans la RID.

Sur la lecture dans les classes populaires on verra SORdi ; SANGa-Rosalio ; SANGa, Lombardia (avec un exemple enregistré sur disque). Sur l'argot et sur la culture des classes de marginaux, je renvoie à SAnga, Gergo ; Sanga, Pastori; SAnga, Cordai ; Bertolotti-Sanga. Des lexiques sont proposés par Prati ; Ferrero.

§ 2. - Dans la rédaction de ce paragraphe, j’ai utilisé mon travail : SANGA, Lettere. Sur l'italien populaire, on verra DE MAURO, Italiano popolare ; SPITZER, Umgangssprache ; SPITZER, Lettere ; Spitzer, Hunger ; Vanelli ; Sobrero, Appunti ; Sobrero, Padroni ; Romanello ; Bellosi ; Rovere, Testi ; Banfi, Storie; et le travail global de Cortelazzo, Lineamenti. Sur le rôle des organisations politico-syndicales ouvrières dans la diffusion de l'alphabétisation, voir Della Peruta ; Becchi ; et la synthèse de Sanga, Modi. Pour les chansons sociales je renvoie au recueil de LEYDI; sur leur importance linguistique, information dans SANGA, Canto. En ce qui concerne les caractéristiques linguistiques des lettres de soldats, je renvoie à Spitzer, Lettere ; SPITZER, Hunger ; SANGA, Lettere ; Bellosi._Les recueils les plus récents de lettres (de soldats, d'émigrés, etc.) sont : Bongiorno-Barbina ; Cheda ; Fontana-Pieretti ; Forcella-Monticone ; Franzina ; Malvezzi-Pirelli ; Raviele ; Revelli.

$\S$ 3. - Pour lïtalien régional se reporter à Pellegrini, Saggi ; Pellegrini, Italiano regionale ; De Mauro, Storia ; Canepari, Introduzione ; CanePari, Presentazione ; et, pour le lexique, à RüEGg. Des monographies régionales, de consistance variée, sont offertes par Llrati ; Bianconi (Tessin); Sanga, Lombardia lavec disque) ; Albanese-ColottiMancarella (Pouilles et Lucanie); Tropea (Sicile). Des monographies sur la province de Bergame (Gaetano BerRLTo, L'Italiano regionale bergamasco), sur la province de Milan (Teresa Poggi Salani), sur le Salento (Maria Teresa Romanello e Alberto A. Sobrero) et sur la Sardaigne (Inès LoI CoRvetTo) sont en préparation. En outre un travail global de Luciano Canepari, Pronunce, est paru.

Pour la norme italienne (standard et régionale) se reporter aux travaux suivants. Phonétique et phonologie : Camilli-Florelli ; Muljaćic, Fonologia ; Canepari, Introduzione ; 
NICH ; Regula-JeRneJ ; HALl, Italiano ; LePSChy-LePSChY (on annonce la publication imminente d'une grammaire de Giulo C. LEPSCHY ; à Padoue, Lorenzo ReNZI et ses collègues ont en préparation une grammaire italienne). Syntaxe : on peut signaler seulement le vieux ForNACIARI ; à Rome Tullio DE MAURo et ses collaborateurs sont en train de préparer une syntaxe italienne. Grammaire historique : RoHLfs, Grammatica ; TeKavčı́c. Graphie : sur les rapports graphie-phonologie, voir FORESTI, Rapporto. (Un premier recensement des graphies des dialectes se trouvera dans la RID 4). Dictionnaires historiques : BatTaglia ; TommaseoBELLINI. Dictionnaires synchroniques : LUI ; ZiNGARELLI (avec transcription phonologique) ; voir en outre le dictionnaire normatif de Migliorini-Tagliavini-Fionelli. Dictionnaires historico-étymologiques : BATTISTI-Alessio ; CoRTelazzo-Zolli. Lexique de fréquence : Bortolini-Tagliavini-Zampolli. Dans les nombreuses études sur l'italien contemporain on peut utilement se reporter à : Migliorini, Lingua contemporanea; Miglionini, Lingua d'oggi ; Devoto-Migliorini-Schiaffini ; Segre ; Peruzzi, Problemi ; Menarini ; BeccaRIA; Italiano d'oggi ; SLI Grammatica trasformazionale ; SLI Sintassi ; SLI Fonetica e fonologia ; SLI Aspetti sociolinguistici ; SLI Insegnamento ; SLI Fenomeni morfologici ; SLI Storia linguistica; Lingua parlata et lingua scritta. Sur la compréhension de l'italien, voir BERRUTO. Sur la standardisation, voir GALLI DE' PARATESI. Les travaux très intéressants pour l'individuation géographique des locuteurs inconnus sont à voir dans TruMPER, Sociolinguistica.

\$ 4. - Sur l'italien bureaucratique, il n'existe pas d'études systématiques. On trouvera des remarques éparses dans DE MAURo, Storia, qui parle aussi de l'italien scolaire ; pour celui-ci, voir BENINCÁ et alii. Le thème a été abordé, même si c'est dans des problématiques différentes, dans le déroulement de la " nouvelle question de la langue " (documents rassemblés par Parlangeli, Questione).

\$ 5. - Sur l'italianisation des dialectes se reporter à De MAUro, Storia ; Trumper, Ricostruzione (formation des koinè régionales) ; SANG A, Lombardia ; Mioni-ArnuZzo LANSZweERT ; Mioni, Situazione (dans lequel on trouve traités les exemples de la Vénétie) ; Foresti, Italianizzazione (exemples bolognais).

\$6. - Pour les ethnicismes linguistiques, je renvoie à Lanternari ; Murru Corriga, Etnicismo ; Sanga, Salvare ; et à l'anthologie de Murru Corriga, Etnia, qui contient des essais notables de Alberto M. Cirese (1969), Giovanni Berlinguer (1975), Giulo ANGioni (1976). On trouvera les positions de Sergio SALVI dans SAlvi, Lingue; celle d'Ulderico Bernardi dans Bernardi, Culture. On verra en outre la synthèse de BANFI, Minoranze; la grande documentation rassemblée dans SLI Minoranze; et le travail à paraître de SANGA, La diffusione degli etnicismi linguistici nell' Italia della crisi.

§ 7. - Dans la rédaction de ce paragraphe, j'ai utilisé en partie SANGA, Lombardia.

Sur la situation sociolinguistique italienne, on verra DE MAuro, Storia; Pellegrini, Saggi ; Sobrero, Padroni ; Sobrero, Appunti ; Mioni, Sociolinguistica ; Mioni-Arnuzzo LANSZwEert ; Mioni, Situazione ; Rosiello, Norma ; et les recueils d'études Bilinguismo e diglossia; Dal dialetto alla lingua ; SLI Minoranze. Sur la sociolinguistique urbaine, voir De Mavro, Città ; Grassi, Comportamento ; Grassi, Ancora. Sur le " continuum " linguistique, voir Mioni-Trumper. Sur l'immigration interne voir SOBRERo, Cambio ; SOBRERo, Interazione. Sur les travailleurs italiens émigrés voir Rovert, Aspetti ; SABÁTINI et alii ; ZaNiER ; TEMPESTA (qui étudie aussi la langue des émigrés de retour au pays). Sur l'émigration stable, voir FranCESCHI ; Rosalio. Des exemples d'enquêtes sociolinguistiques sont présentées par Pautasso (un bourg); Sornicola (un seul locuteur); Giacalone Ramat (enquête avec questionnaire sur une communauté entière de langue allemande) ; SIMONE (enquête avec questionnaire sur un échantillon régional). Pour la sociolinguistique historique, voir TrumPER, Ricostruzione ; SOBRERo, Borgo ; SOBRERo, Modelli. 


\section{RÉFÉRENCES BIBLIOGRAPHIQUES}

AIS : Karl Jaberg-Jakob Jud, Sprach- und Sachatlas Italiens und der Südschweiz [Atlas italosuisse], 8 vol., Zofingen, 1928-1940.

Albanese-Colotti-Mancarella : Elisabetta Albanese, Maria Teresa Colotti, Giovan Battista Mancarella, Italiano regionale in Puglia e Basilicata, Bari, 1979.

Alberoni : Francesco Alberoni "Ormai l'italiano è solo un dialetto europeo, parliamo inglese ", "Corriere della sera ", 23 VII 1978.

ALE : Atlas Linguarum Europae (en préparation, voir SLI Dieci anni, pp. 110-111).

ALEIC : Gino Bottiglioni, Atlante linguistico-etnografico italiano della Corsica, 10 vol., Pisa, 1933-1942.

ALI : Atlante Linguistico Italiano (en préparation, voir SLI Dieci anni, pp. 111-112).

ALM : Atlante Linguistico Mediterraneo (en préparation, voir SLI Dieci anni, p. 110).

ALT : Atlante Lessicale Toscano (en préparation, voir SLI Dieci anni, p. 115).

Arnuzzo-Marcato : Anna Maria Arnuzzo, Gianna Marcato, Lingua e dialetti italiani. Contributo alla bibliografia della lingua e dei dialetti italiani per gli anni 1967-1971, avec la collaboration de Flavia Ursini, Pisa, 1976.

Ascolı, Questione : Graziadio Isaia Ascoli, Scritti sulla questione della lingua, par Corrado Grassi, Milano, 1967 (réédition Torino, 1975).

ASLEF : Giovan Battista Pellegrini, Atlante storico-linguistico-etnografico friulano, plusieurs volumes, Padova-Udine, 1972, en cours (voir SLI Dieci anni, p. 115).

BANfi, Lingua : Emmanuele Banfi, Dalla lingua orale alla lingua scritta, Milano-Firenze, s. d. (1979).

Banfi, Minoranze : Emanuele Banfi, Le lingue delle minoranze etniche, Milano-Firenze, s. d. (1979).

BANFI, Storie : Emanuele Banfi, Analisi linguistica delle * storie personali * : Contributo allo studio dell'italiano popolare, in AA.VV., Pedagogia del linguaggio adulto, par Emanuele Banfi, Milano, 1978.

Battaglia : Salvatore Battaglia, Grande dizionario della lingua italiana, en plusieurs vol., Torino, 1961, en cours.

BatTISTI-Alessio : Carlo Battisti-Giovanni Alessio, Dizionario etimologico italiano, 5 vol., Firenze, 1950-1957.

BECCARIA : I linguaggi settoriali in Italia, par Gian Luigi Beccaria, Milano, 1973.

BECCHI : Egle Becchi, Gli strumenti del leggere e dello scrivere, dans DaLle NoGare.

BELLOSI : Giuseppe Bellosi, Lettere di soldati romagnoli dalle zone di guerra (1915-1918), in RID 3 (1978).

Benincả et alii : Paola Benincà, Giuseppe Ferraboschi, Gianluigi Gaspari, Laura Vanelli, Italiano standard o italiano scolastico?, in Dal dialetto alla lingua (repris dans CoLOMBo).

Bernakid, Culture : Ulderico Bernardi, Le mille culture, Roma, 1976.

BERRUTo : Gaetano Berruto, L'italiano impopolare, Napoli, 1978.

Bertolotti-Sanga : Guido Bertolotti, Felice Bralla, Carlo Butti, Glauco Sanga, I magnani della Val Cavargna e il loro gergo, in Como e il suo territorio, par Roberto Leydi e Glauco Sanga, Milano, 1979.

BiANCONI : Sandro Bianconi, Lingua matrigna, Bologna, 1980.

Bilinguismo e diglossia : Bilinguismo e diglossia in Italia, s. l. (Pisa), s. d. (1973).

BLI : Bibliografia Linguistica Italiana, periodique, Pisa, 1975 et sqq.

Bongionno-Barbina : A. Bongiorno, A. Barbina, Il pane degli altri, lettere di emigranti, Udine, 1970.

Bortolini-Tagliavini-Zampolli : Umberta Bortolini, Carlo Tagliavini, Aldo Zampolli, Lessico di frequenza della lingua italiana contemporanea, Milano, 1972.

Camilli-Fionelli : Amerindo Camilli, Pronuncia e grafia dell'italiano, $3^{e}$ edition, par Piero Fiorelli,, Firenze, 1965.

CANEPARI, Introduzione: Luciano Canepari, Introduzione alla fonetica, Torino, 1979.

Canepari, Presentazione : Luciano Canepari, Presentazione e applicazione allitaliano e alle sue varietà del sistema di trascrizione IPA, in RID 1 (1977). 
CANEPARI, Pronunce : Luciano Canepari, Italiano standard e pronunce regionali, avec deux cassettes, Padova, 1980.

Cardona : Giorgio Raimondo Cardona, Linguistica e politica, in CorsetTI.

Carrannante : Antonio Carrannante, Antonio Gramsci e $i$ problemi della lingua italiana, in "Belfagor 28 (1973).

ChEDA : Giorgio Cheda, L'emigrazione ticinese in Australia, 2 vol., Locarno, 1976.

Colombo : Guida all'educazione linguistica, par Adriano Colombo, Bologna, 1979.

CoRserTI : AA.VV., Lingua e politica, par Renato Corsetti, Roma, 1976.

Cortelazzo, Avviamento : Manlio Cortelazzo, Avviamento critico allo studio della dialettologia italiana. I Problemi e metodi, Pisa, 1969.

Cortelazzo, Lineamenti : Manlio Cortelazzo, Avviamento critico allo studio della dialettologia italiana. III Lineamenti di italiano popolare, Pisa, 1972.

Contelazzo, Profilo : Profilo dei dialetti italiani, collana diretta da Manlio Cortelazzo, plusieurs vol., Pisa, 1974, en cours.

Cortelazzo-Zolli : Manlio Cortelazzo, Paolo Zolli, Dizionario etimologico della lingua italiana, plusieurs vol. :, Bologna, 1979, en cours.

Dal dialetto alla lingua : Dal dialetto alla lingua, Pisa, 1974.

Dalle Nogare : 1815-1898... Quando il popolo cominciò a leggere, par Lilli Dalle Nogare, Milano, 1974.

Della Peruta : Franco Della Peruta, Il * popolo " in Lombardia nell'Ottocento, in Dalle NOGARE.

DE MAURo, Città : Tullio de Mauro, La lingua italiana in città, in “ Il Veltro » 9 (1965).

De Mauro, Italiano popolare : Tullio De Mauro, Per lo studio dell'italiano popolare unitario, in Annabella Rossi, Lettere da una tarantata, Bari, 1970.

De Mauro, Storia : Tullio De Mauro, Storia linguistica dell'Italia unita, 4e edit., Bari, 1974, réédition 1976 (première éd. 1963).

Devoto, Linguaggio : Giacomo Devoto, Il linguaggio d'Italia, Milano, 1974.

Devoto, Profilo : Giacomo Devoto, Profilo di storia linguistica italiana, $4^{\mathrm{e}}$ edit. Firenze, 1964, réimpression 1971 (première ed. 1953).

Devoto-Altieri : Giacomo Devoto, Maria Luisa Altieri, La lingua italiana : storia e problemi attuali, Torino, 1968.

Devoto-Migliorini-Schiaffini : Giacomo Devoto, Bruno Migliorini, Alfredo Schiaffini, Cento anni di lingua italiana, Milano, 1962.

Ferrero : Ernesto Ferrero, I gerghi della malavita dal Cinquecento a oggi, Milano, 1972.

Fiorelli : Piero Fiorelli, Córso di pronùnzia italiana, Padova, 1964.

Fishman : Joshua A. Fishman, La sociologia del linguaggio, Roma, 1975.

Fontana-Pieretti : La Grande Guerra, Operai e contadini lombardi nel primo conflitto mondiale, par Sandro Fontana et Maurizio Pieretti, Milano, 1980.

Forcella-Monticone : Enzo Forcella, Alberto Monticone, Plotone d'esecuzione : $i$ processi della prima guerra mondiale, Bari, 1968.

Foresti, Italianizzazione : Fabio Foresti, Cenni sull'italianizzazione del dialetto bolognese con particolare riferimento all'aspetto lessicale, in Dal dialetto alla lingua.

Foresti, Rapporto : Fabio Foresti, Il rapporto tra sistemi grafici e sistemi fonologici, con particolare riguardo all'italiano, in RID 1 (1977).

FORNACIARI : Raffaello Fornaciari, Sintassi italiana dell'uso moderno, réimpression, Firenze, 1974 (première éd. 1881 ).

Franceschi : Temistocle Franceschi, Lingua e cultura di una communità italiana in Costa Rica, Firenze, 1970.

Franzina : Emilio Franzina, Merica ! Merica ! Emigrazione e colonizzazione nelle lettere dei contadini veneti in America Latina 1876-1902, Milano, 1979.

Galli DE' Paratesi : Nora Galli De' Paratesi, La standardizzazione nella pronuncia dell'italiano contemporaneo, in SLI. Aspetti sociolinguistici.

Giacalone Ramat : Anna Giacalone Ramat, Lingua, dialetto e comportamento linguistico. La situazione di Gressoney, Aosta, 1979.

GiaCalone Ramat : Anna Giacalone Ramat, Lingua, dialetto e comportamento linguistico. La Bologna, 1967 (première éd., 1918). 
Gramsci : Antonio Gramsci, Quaderni del carcere, éd. critique par Valentino Gerratana, Torino, 1975.

Grassi, Ancora : Corrado Grassi, Ancora su * Comportamento linguistico e comportamento sociologico », in AGI 50 (1965).

GraSSI, Comportamento : Corrado Grassi, Comportamento linguistico e comportamento sociologico, in AGI 49 (1964).

Hall, Bibliografia : Robert A. Hall jr., Bibliografia della linguistica italiana, $2^{e}$ édit., 3 vol., Firenze, 1958.

Hall, Bibliografia essenziale : Robert A. Hall jr., Bibliografia essenziale della linguistica italiana e romanza, Firenze, 1973.

HALl, Italiano : Robert A. Hall jr., La struttura dell'italiano, Roma, 1971.

HALL, Supplemento : Robert A. Hall jr., Bibliografia della linguistica italiana. Primo supplemento decennale (1956-1966), Firenze, 1969.

Italiano d'oggi : Italiano d'oggi, Trieste, 1974.

LANTERNARI : Vittorio Lanternari, Crisi e ricerca d'identità. Folklore e dinamica culturale, $2^{e}$ éd., Napoli, 1977.

LEPSCHY : Giulio C. Lepschy, Saggi di linguistica italiana, Bologna, 1978.

LEPSCHY-LEPSCHY : Anna Laura Lepschy, Giulio C. Lepschy, The Italian Language Today, London, 1977.

LEYDI : Roberto Leydi, Canti sociali italiani, Milano, 1963.

Lichem : Klaus Lichem, Phonetik und Phonologie des heutigen Italienisch, München, 1969.

Lingua parlata e lingua scritta : Lingua parlata e lingua scritta, in "Bollettino del Centro di Studi Filologici e Linguistici Siciliani ", 11 (1970).

Lo Piparo : Franco Lo Piparo, Lingua intellettuali egemonia in Gramsci, Roma-Bari, 1979.

LUI : Istituto dell'Enciclopedia Italiana, Lessico Universale Italiano, 25 vol., Roma, 1969 et sqq.

Lurati : Ottavio Lurati, Dialetto e italiano regionale nella Svizzera Italiana, Lugano, 1976.

Malagoli : G. Malagoli, L'accentazione italiana. Guida pratica, Firenze, 1946, $2^{\mathrm{e}}$ éd. 1968.

Malvezzi-Pirelli : Lettere di condannati a morte della Resistenza italiana (8 settembre 1943-25 aprile 1945), par Piero Malvezzi et Giovanni Pirelli, Torino, 1961.

Marcellesi-Ponzio : Jean-Baptiste Marcellesi et alii, Linguaggio e classi sociali. Marrismo e stalinismo, introduction d'Augusto Ponzio, Bari, 1978.

Menarini : Alberto Menarini, Ai margini della lingua, Firenze, 1947.

Migliorini, Lingua contemporanea : Bruno Migliorini, Lingua contemporanea, $4^{\mathrm{e}}$ éd., Firenze, 1963.

Migliorini, Lingua d'oggi : Bruno Migliorini, La lingua italiana d'oggi, $2^{\mathbf{e}}$ ed., Torino, 1967.

Migliorini, Questione : Bruno Migliorini, La questione della lingua, in Problemi e orientamenti critici di lingua e letteratura italiana, par Attilio Momigliano, vol. III, Milano, 1949.

Migliorini, Storia : Bruno Migliorini, Storia della lingua italiana, Firenze, 1960.

Migliorini-Baldelli : Bruno Migliorini, Ignazio Baldelli, Breve storia della lingua italiana, Firenze, 1964.

Migliorini-Tagliavini-Fiorelli : Bruno Migliorini, Carlo Tagliavini, Piero Fiorelli, Dizionario d'ortografia e pronuncia, Torino, 1969, $2^{e}$ éd., 1970.

MIONI, Situazione : Alberto M. Mioni, La situazione sociolinguistica italiana : lingua, dialetti, italiani regionali, in Colombo.

MIONI, Sociolinguistica : Alberto M. Mioni, Per una sociolinguistica italiana. Note di un non sociologo, in FishMAN.

Mioni-Arnuzzo Lanszweert : Alberto M. Mioni, Anna Maria Arnuzzo Lanszweert, Sociolinguistics in Italy, in "International Journal of the Sociology of Language" 21 (1979).

Mroni-Trumper : Alberto M. Mioni-John Trumper, Per un'analisi del "continuum " linguistico veneto, in SLI Aspetti sociolinguistici.

MONTEleone : Renato Monteleone, Lettere al re, Roma, 1973.

MulJacić, Fonologia : Zarko Muljačić, Fonologia generale e fonologia della lingua italiana, Bologna, 1969. 
MULJAČIĆ, Introduzione : Žarko Muljačić, Introduzione allo studio della lingua italiana, Torino, 1971.

Murru Corriga, Etnia : Etnia lingua cultura : un dibattito aperto in Sardegna, par Giannetta Murru Corriga, Cagliari, 1977.

MurRu Corriga, Etnicismo : Giannetta Murru Corriga, Etnicismo e cultura popolare nel dibattito recente in Sardegna. Primi appunti per un'analisi, in " BRADS. Bollettino del repertorio dell'Atlante demologico sardo " 8 (1977-78).

Parlangéli, Bibliografia: Oronzo Parlangèli, Bibliografia dialettale italiana (1962-1966), in * Bollettino della Carta dei Dialetti Italiani " 1 (1966).

Parlangèlt, Questione : La nuova questione della lingua, essais recueillis par O. Parlangèli, Brescia, 1971.

Parlangèli, Saggio : Oronzo Parlangèli, Saggio di una bibliografia dialettale italiana (1955-1962), in "Italia Dialettale » 25 (1962) et 26 (1963) (et aussi en volume, Pisa, 1964).

Pautasso: Mariella Pautasso, Dialetto, lingua $e$ integrazione linguistica a Pettinengo, Torino, 1969.

Pellegrini, Carta : Giovan Battista Pellegrini, Carta dei dialetti italiani, 1 vol. et 1 carte, Pisa, 1977.

Pellegrini, Italiano regionale : Giovan Battista Pellegrini, L'italiano regionale, in "Cultura e scuola " 2 (1963).

Pellegrini, Saggi : Giovan Battista Pelligrini, Saggi di linguistica italiana, Torino, 1975.

Peruzzi, Lingua : Emilio Peruzzi, Una lingua per gli italiani, Torino, 1964, $2^{e}$ éd. 1967.

Peruzzi, Problemi : Emilio Peruzzi, Problemi di grammatica italiana, Torino, 1960.

Prati : Angelico Prati, Voci di gerganti, vagabondi $e$ malviventi studiate nell'origine $e$ nella storia, Pisa, 1940 (réimpression Pisa, 1979).

RAVIELE : Lettere dall'Itaglia. Lettere di soldati meridionali dai fronti della Grande Guerra, par Gianni Raviele, Napoli, 1977.

Regula-JerneJ : M. Regula, J. Jernej, Grammatica italiana descrittiva su basi storiche e psicologiche, Bern-München, 1965.

RID : " Rivista italiana di dialettogia. Scuola società territorio ", Bologna, 1977, en cours.

RoHlfs, Grammatica : Gerhard Rohlfs, Grammatica storica della lingua italiana e dei suoi dialetti, 3 vol., Torino, 1966-1969.

RoHLFS, Studi : Gerhard Rohlfs, Studi e ricerche su lingua e dialetti d'Italia, Firenze, 1972.

Romanello : Maria Teresa Romanello, "Una scrittura di classe ", in "Sigma " 2-3 (1978).

Rosalio : Maria Rita Rosalio, Studi sul dialetto trentino di Stivor (Bosnia), Firenze, 1979.

Rosiello, Gramsci : Luigi Rosiello, Problemi linguistici negli scritti di Gramsci, in Gramsci e la cultura contemporanea, vol. II, Roma, 1970.

Rosiello, Norma : Luigi Rosiello, Norma, dialetto e diasistema dell'italiano regionale, in SLI Insegnamento.

REVELLI : Nuto Revelli, L'ultimo fronte : lettere di soldati caduti o dispersi nella seconda guerra mondiale, Torino, 1971.

ROVERE, Emigrazione : Giovanni Rovere, Aspetti sociolinguistici dell'emigrazione italiana in Svizzera, in "Vox romanica " 33 (1974).

Rovere, Testi : Giovanni Rovere, Testi di italiano popolare, Roma, 1977.

RUEGG : R. Rüegg, Zur Wortgeographie der italienischen Umgangssprache, Köln, 1956.

Sabatini et alii : Francesco Sabatini, Luigi M. Lombardi Satriani, Raffaele Simone, Lingua italiana e processi di acculturazione in Europa (considerazioni sullo status internazionale dell'italiano), in Italiano d'oggi.

SALVI, Lingue : Sergio Salvi, Le lingue tagliate, Milano, 1975.

SANGA, Canto : Glauco Sanga, Il linguaggio del canto popolare, avec deux cassettes, MilanoFirenze, s.d. (1979).

SANGA, Cordai : Glauco Sanga, I cordai di Castelponzone. Da "dritti a proletari, in Cremona e il suo territorio, par Roberto Leydi et Guido Bertolotti, Milano, 1979.

SAngA, Dialetto : Glauco Sanga, Il dialetto. Note di linguistica materialista, in RID 1 (1977).

SANGA, Gergo : Glauco Sanga, Il gergo e il rapporto lingua-classe, in SLI Minoranze.

SANGA, Lettere : Glauco Sanga, Lettere dei soldati e formazione dell'italiano popolare unitario, in Fontana-Pieretti. 
SANGA, Lombardia : Glauco Sanga, La situazione linguistica in Lombardia, avec un disque, in Il paese di Lombardia, Milano, 1978.

SANGA, Modi : Glauco Sanga, Modi di produzione e forme di tradizione : dall'oralità feudale alla scrittura capitalistica, communication au Congrès "Oralità e scrittura nel sistema letterario "(Cagliari, 1980), à paraître dans les Actes.

SANGA, Pastori : Glauco Sanga, Il gergo dei pastori bergamaschi, in Bergamo e il suo territorio, par Roberto Leydi, Milano, 1977.

Sanga, Principii : Glauco Sanga, Principii di linguistica materialista, communication au Congrès SLI "I Presupposti ideologici delle ricerche linguistiche " (Cosenza, 1978), à paraître dans les Actes.

SANGA, Salvare : Glauco Sanga, Salvare il dialetto?, in "BC. Beni culturali " 2 loctobredécembre 1978).

SANGA, Trascrizione : Glauco Sanga, Sistema di trascrizione semplificato secondo la grafia italiana, in RID 1 (1977).

SANGa-Rosalio : Glauco Sanga, dans Rita Rosalio, Un lettore popolare, in Brescia e il suo territorio, par Roberto Leydi e Bruno Pianta, Milano, 1976.

SEGRE : Cesare Segre, Le caratteristiche della lingua italiana, in Charles Bally, Linguistica generale e linguistica francese, Milano, 1963.

SIMONE : Raffaele Simone, L'educazione linguistica per gli adulti, Roma, 1976.

SLI Aspetti sociolinguistici : Società di Linguistica Italiana, Aspetti sociolinguistici dell'Italia contemporanea, par Kaffaele Simone et Giulianella Ruggiero, 2 vol., Roma, 1977.

SLI Dieci anni : Società di Linguistica Italiana, Dieci anni di linguistica italiana (19651975), par Daniele Gambarara et Paolo Ramat, Roma, 1977.

SLI Fenomeni morfologici : Società di Linguistica Italiana, Fenomeni morfologici e sintattici nell'italiano contemporaneo, par Mario Medici et Antonella Sangregorio, 3 vol., Roma, 1974.

SLI Fonetica e fonologia : Società di Linguistica Italiana, Studi di fonetica e fonologia, par Raffaele Simone, Ugo Vignuzzi et Giulianella Ruggiero, Roma, 1976.

SLI Grammatica trasformazionale : Società di Linguistica Italiana, Grammatica trasformazionale italiana, par Mario Medici e Raffaele Simone, Roma, 1971.

SLI Insegnamento : Società di Linguistica Italiana, L'insegnamento dell'italiano in Italia e all'estero, par Mario Medici e Raffaele Simone, 2 vol., Roma, 1971.

SLI Minoranze : Società di Linguistica Italiana, $I$ dialetti $e$ le lingue delle minoranze di fronte all'italiano, par Federico Albano Leoni, 2 vol., Roma, 1980.

SLI Sintassi : Società di Linguistica Italiana, La sintassi, par Wanda D'Addio e Raffaele Simone, Roma, 1970.

SLI Storia linguistica : Società di Linguistica Italiana, Storia linguistica dell'Italia nel Novecento, par Maurizio Gnerre, Mario Medici et Raffaele Simone, Roma, 1973.

Sobrero, Appunti : Alberto Sobrero, Una società fra dialetto e lingua. Appunti di italiano contemporaneo, Lecce, 1974.

SoBRERo, Borgo : Alberto A. Sobrero, Borgo, città, territorio : alcuni problemi di metodo nella dialettologia urbana, in RID 2 (1978).

Sobrero, Cambio : Alberto Sobrero, Il cambio linguistico nell'acculturazione dell'immigrato : nuovi problemi di glottodidattica, in Bilinguismo e diglossia.

SOBRERo, Integrazione: Alberto Sobrero, L'integrazione linguistica in giovani immigrati a Torino, in * Parole e Metodi * 6 (octobre 1973).

SOBRERo, Modelli : Alberto A. Sobrero, Modelli sociolinguistici e modelli storici, in Questioni di confine, «Quaderni storici » 40 (janvier-avril 1979).

Sobrero, Padroni : Alberto A. Sobrero, I padroni della lingua. Profilo sociolinguistico della lingua italiana, Napoli, 1978.

SORDI : Italo Sordi, Gli strumenti linguistici della cultura orale lombarda, in Il paese di Lombardia, Milano, 1978.

SORNICOLA : Rosanna Sornicola, La competenza multipla. Un'analisi micro-socio-linguistica, Napoli, 1977.

SozzI : Bortolo Tommaso Sozzi, Aspetti e momenti della questione linguistica, Padova, 1955.

SPITZER, Hunger : Leo Spitzer, Die Umschreibungen des Begriffes "Hunger * im Italienischen, Halle, 1920. 
SPITZER, Lettere : Leo Spitzer, Lettere di prigionieri di guerra italiani 1915-1918, Torino, 1976.

SPITZER, Umgangssprache : Leo Spitzer, Italienische Umgangssprache, Bonn-Leipzig, 1922.

STUSSI : Alfredo Stussi, Lingua, dialetto e letteratura, in Storia d'Italia. 1. I caratteri originali, Torino, 1972.

Sylos Labini : Paolo Sylos Labini, Saggio sulle classi sociali, Bari, 1974, 5e éd., 1975.

Tagliavini, Origini : Carlo Tagliavini, Le origini delle lingue neolatine, $5^{\mathrm{e}}$ éd., Bologna, 1969 (1 ${ }^{\text {re }}$ éd. 1949).

Tagliavini, Pronuncia : Carlo Tagliavini, La corretta pronuncia italiana. Corso discografico di fonetica e ortoepia, Bologna, 1965.

TeKavČIC : Pavao Tekavčić, Grammatica storica dell'italiano, 3 vol., Bologna, 1972.

Tempesta : Immacolata Tempesta, Lingua ed emigrazione, Lecce, 1978.

Terracini-Franceschi : Benvenuto Terracini, Temistocle Franceschi, Saggio di un atlante linguistico della Sardegna, Torino, 1964.

Tommaseo-Bellini : Nicolò Tommaseo, Bernardo Bellini, Dizionario della lingua italiana, 4 vol., Torino, 1858-1879, réimpression en 20 vol., Milano, 1977.

Tropea : Giovanni Tropea, Italiano di Sicilia, Palermo, 1976.

TruMPER, Ricostruzione: John Trumper, Ricostruzione nell'Italia settentrionale : sistemi consonantici. Considerazioni sociolinguistiche nella diacronia, in Società di Linguistica Italiana, La ricostruzione linguistica, par Raffaele Simone e Ugo Vignuzzi, Roma, 1977.

Trumper, Sociolinguistica : John Trumper, Sociolinguistica giudiziaria, Padova, 1979.

VANELli : Laura Vanelli, Nota linguistica, in SPITZER, Lettere.

Vitale, Questione : Maurizio Vitale, La questione della lingua, nouvelle édition augmentée, Palermo, 1978 (1 $1^{\text {re }}$ ed. 1960 ).

ZANIER : La lingua degli emigrati, par L. Zanier, Rimini-Firenze, 1977. 